\title{
Phase diagram of dense two-color QCD within lattice simulations
}

\author{
V.V. Braguta ${ }^{1,2,3,4, a}$, E.-M. Ilgenfritz ${ }^{5, b}$, A.Yu. Kotov ${ }^{2,6, c}$, A.V. Molochkov ${ }^{3, d}$, and A.A. Nikolaev ${ }^{2,3, e}$ \\ ${ }^{1}$ Institute for High Energy Physics NRC "Kurchatov Institute", Protvino, 142281 Russia \\ ${ }^{2}$ Institute for Theoretical and Experimental Physics NRC "Kurchatov Institute", Moscow, 117218 Russia \\ ${ }^{3}$ School of Biomedicine, Far Eastern Federal University, Sukhanova 8, Vladivostok, 690950 Russia \\ ${ }^{4}$ Moscow Institute of Physics and Technology, Institutskii per. 9, Dolgoprudny, Moscow Region, 141700 \\ Russia \\ ${ }^{5}$ Joint Institute for Nuclear Research, BLTP, Dubna, 141980 Russia \\ ${ }^{6}$ National Research Nuclear University MEPhl (Moscow Engineering Physics Institute), Kashirskoe Highway, \\ 31, Moscow 115409, Russia
}

\begin{abstract}
We present the results of a low-temperature scan of the phase diagram of dense two-color QCD with $N_{f}=2$ quarks. The study is conducted using lattice simulation with rooted staggered quarks. At small chemical potential we observe the hadronic phase, where the theory is in a confining state, chiral symmetry is broken, the baryon density is zero and there is no diquark condensate. At the critical point $\mu=m_{\pi} / 2$ we observe the expected second order transition to Bose-Einstein condensation of scalar diquarks. In this phase the system is still in confinement in conjunction with nonzero baryon density, but the chiral symmetry is restored in the chiral limit. We have also found that in the first two phases the system is well described by chiral perturbation theory. For larger values of the chemical potential the system turns into another phase, where the relevant degrees of freedom are fermions residing inside the Fermi sphere, and the diquark condensation takes place on the Fermi surface. In this phase the system is still in confinement, chiral symmetry is restored and the system is very similar to the quarkyonic state predicted by $S U\left(N_{c}\right)$ theory at large $N_{c}$.
\end{abstract}

\section{Introduction.}

The phase diagram of QCD is of high importance for several fields of observational physics like cosmology and astrophysics. One field of experimental physics, located between nuclear physics and high energy physics, is the study of hadronic matter created by relativistic heavy ion collisions. Such experiments are addressing the structure of the phase diagram, although the understanding and modeling of an actual collision requires much more than the knowledge of the equilibrium phase diagram. However, equilibrium observables like the equation of state and transport coefficients are highly needed to be used in hydrodynamical approaches which serve to probe various scenarios.

\footnotetext{
ae-mail: braguta@itep.ru

be-mail: ilgenfri@theor.jinr.ru

ce-mail: kotov@itep.ru

de-mail: molochkov.av@dvfu.ru

e-mail: nikolaev.aa@dvfu.ru
} 
Unfortunately, lattice simulation of QCD can not be applied today to arbitrary chemical potential because of the sign problem [1]. The origin of the sign problem is that the fermion determinant becomes complex-valued which makes impossible the direct simulation by importance sampling of gauge field configurations. An alternative to lattice simulation of $\operatorname{SU}(3)$ QCD with $\mu \neq 0$ is the simulation of $\mathrm{SU}(2) \mathrm{QCD}$ (also called $\mathrm{QC}_{2} \mathrm{D}$ ). Introduction of a chemical potential to the latter theory does not lead to a sign problem, so one can apply the standard lattice approach to study this theory. Although a two-color world differs from the tree-color world, lattice study of $\mathrm{QC}_{2} \mathrm{D}$ with chemical potential can provide us with important information about the properties of QCD with non-zero baryon density (EoS, generation of the fermion mass gap, etc.). We would like also to note that it is interesting to study the $\mathrm{QC}_{2} \mathrm{D}$ by its own due to a rich structure of the phase diagram.

The properties of $\mathrm{QC}_{2} \mathrm{D}$ were studied theoretically within the following approaches: ChPT [24], the NJL model [5-7], FRG [8, 9], random matrix theory [10-12]. Principally, these studies have revealed the following phase structure of low temperature $\mathrm{QC}_{2} \mathrm{D}$ with three subsequent phases: (1) $0<\mu<\mu^{c}$ (hadronic phase), (2) $\mu^{c}<\mu<\mu^{d}$ ("baryon onset" with a superfluid condensate due to Bose-Einstein condensation [BEC]) and (3) $\mu^{d}<\mu$ (the phase with diquark condensation due to the Bardeen-Cooper-Schrieffer mechanism [BCS] [13]).

The first lattice study of $\mathrm{QC}_{2} \mathrm{D}$ with chemical potential and Wilson fermions was performed by A. Nakamura in [14]. Futher lattice investigation of dense two-color QCD was continued by J. Kogut and collaborators using staggered quarks. In [15] eight-flavor theory was investigated and in [16-18] the authors studied $N_{f}=4$ theory. What concerns a low temperature scan of the phase diagram, these authors observed the succession of a hadronic phase and the BEC phase, with their properties well described by ChPT, but they didn't find a BCS phase.

The main activity in two-color QCD was later continued by the Swansea group (S. Hands and collaborators) for the two-flavor theory with Wilson fermions [19-22]. In a low temperature scan of the phase diagram the authors observed a hadronic phase, followed by the BCS phase with deconfinement, but did not encounter the BEC phase.

In this paper we are going to study the $\mathrm{QC}_{2} \mathrm{D}$ phase diagram with $N_{f}=2$ flavors going back to the lattice simulation of staggered fermions using the rooting procedure. In the present paper we are going to carry out a $\mu^{1}$ scan at low temperature of the $\mathrm{QC}_{2} \mathrm{D}$ phase diagram.

\section{The lattice set-up}

In our simulations we used the Wilson action for the $\mathrm{SU}(2)$ gauge fields

$$
S_{G}=\beta \sum_{x} \sum_{\mu<v=1}^{4}\left(1-\frac{1}{2} \operatorname{Tr} U_{x, \mu v}\right) .
$$

For the fermionic degrees of freedom we used staggered fermions with an action of the form

$$
\begin{gathered}
S_{F}=\sum_{x, y} \bar{\psi}_{x} M(\mu, m)_{x, y} \psi_{y}+\frac{\lambda}{2} \sum_{x}\left(\psi_{x}^{T} \tau_{2} \psi_{x}+\bar{\psi}_{x} \tau_{2} \bar{\psi}_{x}^{T}\right) \\
M_{x y}=m a \delta_{x y}+\frac{1}{2} \sum_{\mu=1}^{4} \eta_{\mu}(x)\left[U_{x, \mu} \delta_{x+\hat{\mu}, y} e^{\mu a \delta_{\mu, 4}}-U_{x-\hat{\mu}, \mu}^{\dagger} \delta_{x-\hat{\mu}, y} e^{-\mu a \delta_{\mu, 4}}\right],
\end{gathered}
$$

\footnotetext{
${ }^{1}$ Quark chemical potential is understood by $\mu$ here and below.
} 
where $\bar{\psi}, \psi$ are staggered fermion fields, $a$ is the lattice spacing, $m$ is the bare quark mass, and $\eta_{\mu}(x)$ are the standard staggered phase factors: $\eta_{1}(x)=1, \eta_{\mu}(x)=(-1)^{x_{1}+\ldots+x_{\mu-1}}, \mu=2,3,4$. The chemical potential $\mu$ is introduced into equation (3) through the multiplication of the links along and opposite to the temporal direction by factors $e^{ \pm \mu a}$ respectively. This way of introducing the chemical potential makes it possible to avoid additional divergences and to reproduce well known continuum results [23].

In addition to the standard staggered fermion action we add a diquark source term [15] to equation (2). The diquark source term explicitly violates $U_{V}(1)$ and allows to observe diquark condensation even on finite lattices. The results presented in this paper are obtained as follows: we carry out simulations at small but nonzero parameter $\lambda \ll m a$, and then extrapolate obtained data to $\lambda \rightarrow 0$. Notice that similar to the diquark source term an additional pion term was introduced to the fermion action during the studies of QCD phase diagram with isospin chemical potential [24-26].

Integrating out the fermion fields the partition function for the theory can be written as

$$
Z=\int D U e^{-S_{G}} \cdot P f\left(\begin{array}{cc}
\lambda \tau_{2} & M \\
-M^{T} & \lambda \tau_{2}
\end{array}\right)=\int D U e^{-S_{G}} \cdot \sqrt{\operatorname{det}\left(M^{\dagger} M+\lambda^{2}\right)}
$$

where $M$ is the staggered Dirac operator and $\lambda$ is the diquark source. Note that the pfaffian $P f$ is strictly positive, such that one can use Monte-Carlo methods to study this system. Partition function (4) corresponds to $N_{f}=4$ in the continuum limit. In the present paper we are going to study

$$
Z=\int D U e^{-S_{G}} \cdot\left[\operatorname{det}\left(M^{\dagger} M+\lambda^{2}\right)\right]^{1 / 4}
$$

which corresponds to the theory with $N_{f}=2$ dynamical fermions in the continuum limit.

To study the phase diagram of $\mathrm{QC}_{2} \mathrm{D}$ with $N_{f}=2$ flavors we used a $16^{3} \times 32$ lattice, simulating with $\beta=2.15, m a=0.005$, the lattice spacing $a=0.112(1) \mathrm{fm}$ and the pion mass $M_{\pi}=378(4) \mathrm{MeV}$ (detailed description of the action and parameters may be found in [27]). The simulation was carried out for a set of values of the chemical potential $\mu$ spanning the region $\mu \in[0 ; 1759] \mathrm{MeV}$ ( $\mu a \in$ $[0.0 ; 1.0])$. For each value of $\mu \in[0 ; 1055] \mathrm{MeV}(\mu a \in[0.0 ; 0.6])$ we carried out the simulation at three values of the diquark source: $\lambda=0.001,0.00075$ and 0.0005 , the measured data have been then extrapolated to $\lambda=0$. Simulations with higher $\mu$ are more computationally demanding, thus for $\mu>1055 \mathrm{MeV}(\mu a>0.6)$ only the value $\lambda=0.0005$ was used.

\section{Numerical results}

\subsection{The diquark condensate}

In Fig. 1 we plot the diquark condensate $\langle q q\rangle$, obtained by linear extrapolation to $\lambda=0$, as a function of $\mu$ in the region $\mu \in[0.0 ; 440] \mathrm{MeV}(\mu a \in[0.0 ; 0.25])$. It may be seen, that for $\mu \leq 141 \mathrm{MeV}$ $(\mu a \leq 0.08)$ the diquark condensate $\langle q q\rangle$ is compatible with zero, i.e. the system is in the hadronic phase. However, for $\mu \geq 176 \mathrm{MeV}(\mu a \geq 0.1)$ the diquark condensate starts to deviate from zero. If we are sufficiently far from the position of the phase transition, one can try to use ChPT [2, 3] to describe the data. In particular, ChPT predicts that at $\mu^{c}=m_{\pi} / 2$ there is the transition from the hadronic phase to the phase of Bose-Einstein condensation of scalar diquarks with $\langle q q\rangle \neq 0$, and the behaviour of the diquark condensate above the transition would be given by the formula $\langle q q\rangle=\langle\bar{q} q\rangle_{0} \sqrt{1-\left(\mu^{c} / \mu\right)^{4}}$, where $\langle\bar{q} q\rangle_{0}$ is the chiral condensate at zero chemical potential. Fit with this formula in the region $\mu \in[263 ; 352] \mathrm{MeV}(\mu a \in[0.15 ; 0.20])$ provides $\mu^{c}=215(10) \mathrm{MeV}$ $\left(a \mu^{c}=0.122(6)\right)$ with $\chi^{2} / d o f=2.5$. We plot this fit in the Fig. 1. 


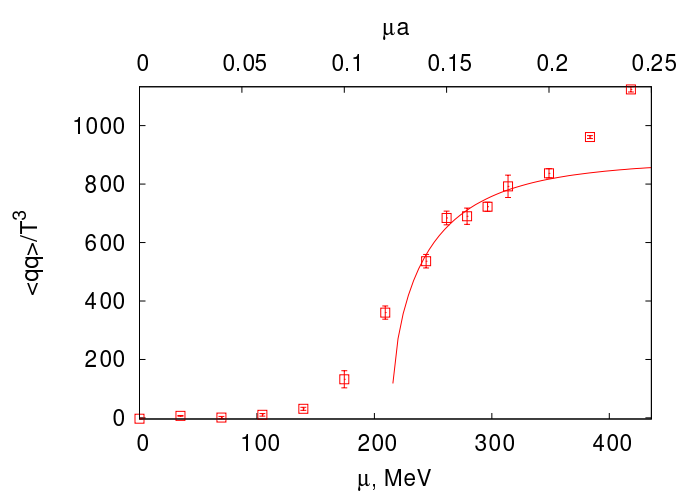

Figure 1. The diquark condensate as a function of $\mu$.

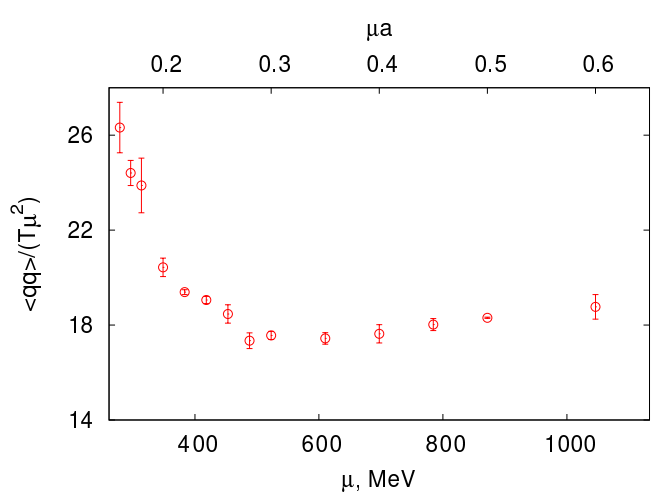

Figure 2. The ratio $\langle q q\rangle /\left(T \mu^{2}\right)$ as a function of $\mu$.

One can try to fit the data by another function: $\langle q q\rangle=\langle\bar{q} q\rangle_{0} \sqrt{1-\left(\mu^{c} / \mu\right)^{2 \alpha}}$ with the power $\alpha$ considered as an additional fitting parameter. The fit with this formula in the same region $\mu \in[263 ; 352]$ $\mathrm{MeV}(\mu \in[0.15,0.20])$ gives $\mu^{c}=193(10) \mathrm{MeV}\left(a \mu^{c}=0.110(6)\right)$ with $\chi^{2} / d o f=1.4$. From these examples one sees, that the position of the critical point determined from the fitting procedure strongly depends on the fitting function. Nevertheless, the results for $\mu^{c}$ and the behaviour of $\langle q q\rangle$ are in reasonable agreement with $\mathrm{ChPT}$ predictions in the region $\mu a \in[0.0 ; 0.20]$.

Let us consider the region of larger chemical potential $\mu>352 \mathrm{MeV}(\mu a>0.2)$. To understand what happens in this region, we plot in Fig. 2 the linearly extrapolated diquark condensate, divided by $T \mu^{2}$, as a function of $\mu$. As it is visible from this plot, in the region $\mu \in[528 ; 1055] \mathrm{MeV}$ ( $\mu a \in$ $[0.3 ; 0.6])$ there is a plateau, i.e. the value of the diquark condensate is proportional to the surface of a sphere with the radius $\mu$ : $\langle q q\rangle \sim \mu^{2}$. This is a characteristic property of the BCS theory, where the condensate appears on the Fermi surface and is proportional to the density of states on this surface. Thus we conclude, that for $\mu>528 \mathrm{MeV}(\mu a>0.3$ ) the system reveals properties of the BCS phase, and that the transition from the BEC to the BCS phase is smooth.

\subsection{The chiral condensate}

In the Fig. 3 we plot the chiral condensate calculated for the smallest diquark source value $\lambda=0.0005$ as a function of $\mu$ (during the simulations we discovered that dependence of the chiral condensate on the source $\lambda$ was very weak). The first observation is that up to $\mu<176 \mathrm{MeV}(\mu a<0.1)$, which is approximately $m_{\pi} / 2$, the chiral condensate does not depend on the chemical potential. In the region $\mu>176 \mathrm{MeV}$, where the system is in the vicinity of the transition to the BEC phase, the chiral condensate starts to decrease. These properties are in agreement with ChPT predictions (see the Figures 4 and 5 in paper [2]).

According to ChPT, at $\mu>\mu^{c}$ the chiral condensate drops as $\langle\bar{q} q\rangle=\langle\bar{q} q\rangle_{0}\left(\mu^{c} / \mu\right)^{2}$. To check this prediction in the region $\mu \in[263 ; 352] \mathrm{MeV}(\mu a \in[0.15 ; 0.20])$ we fitted our data by a power law $\langle\bar{q} q\rangle=A / \mu^{\alpha}$, which provided $\alpha=0.78(2)$ with $\chi^{2} / d o f=0.3$. It is interesting to note, that this fit gives a satisfactory description of the data up to $\mu \sim 1055 \mathrm{MeV}(\mu a \sim 0.6)$. Thus, one sees that the chiral condensate drops slower with increasing chemical potential than ChPT predicts (similar slower decrease of the form $\langle\bar{q} q\rangle \sim 1 / \mu$ was observed in [21] with $N_{f}=2$ Wilson quarks). 


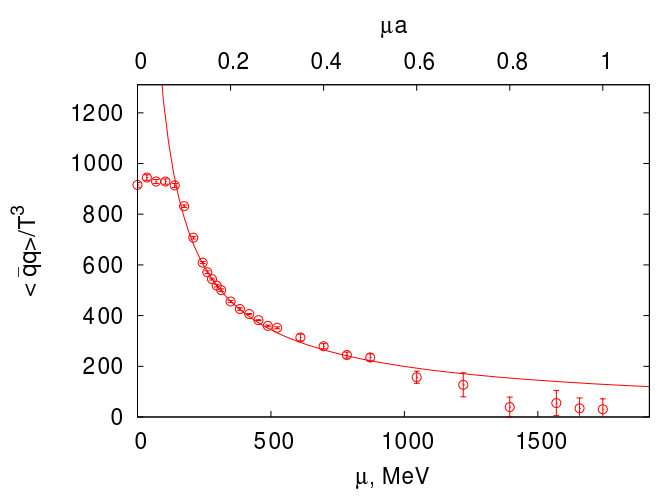

Figure 3. The chiral condensate $\langle\bar{q} q\rangle / T^{3}$ as a function of $\mu$.

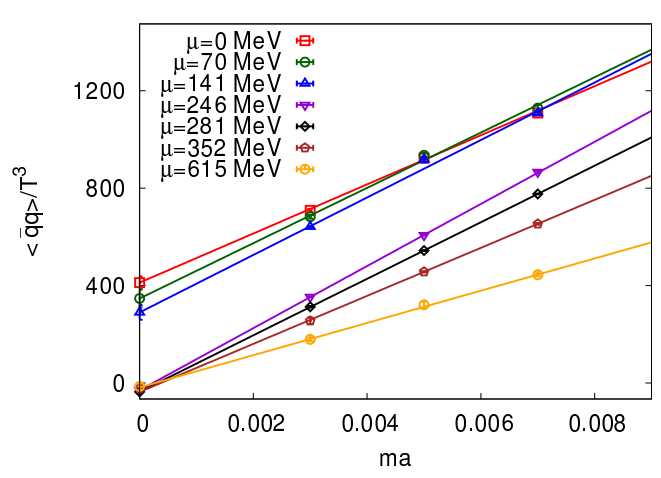

Figure 4. The chiral limit of the chiral condensate taken for different values of the chemical potential.

Finally, it is interesting to study the chiral symmetry breaking in the chiral limit for different regions of the chemical potential. In Fig. 4 we plot the chiral condensate for different values of the chemical potential as a function of the quark mass and the results of the linear extrapolation to $m a=0$. It may be seen from Fig. 4, that chiral symmetry breaking exists in the chiral limit within the hadronic phase (values $\mu=0,70$ and $141 \mathrm{MeV}$ ), whereas there is no chiral symmetry breaking in the chiral limit in the BCS phase $(\mu=615 \mathrm{MeV})$ and in the BEC phase $(\mu=246,281,352 \mathrm{MeV})$. However, it is difficult to claim, that there is no chiral symmetry breaking in the whole BEC phase: when we take the chiral limit, we change the pion mass and thus shift the critical point $\mu^{c}$ closer to zero. This effect might be important near the phase transition. Note, that the absence of chiral symmetry breaking in the chiral limit within the BEC phase agrees with ChPT predictions.

\subsection{The baryon density}

In Fig. 5 we plot the baryon density ${ }^{2}$ in the region $\mu \in[0.0 ; 528] \mathrm{MeV}(\mu a \in[0.0 ; 0.3])$. It is clear, that for all $\mu<176 \mathrm{MeV}(\mu a<0.1)$ the baryon density is vanishing within the uncertainty of the calculations. In the vicinity of the phase transition $(\mu \geq 176 \mathrm{MeV})$ the baryon density starts to deviate from zero, and for larger values of the chemical potential it rises with the increasing of $\mu$. ChPT predicts, that the dependence of the baryon density on the chemical potential above $\mu^{c}$ is given by a formula $n_{B} \sim \mu-\mu_{c}^{4} / \mu^{3}$. Fit of the data by this formula in the region $\mu \in[263 ; 352] \mathrm{MeV}$ $(\mu a \in[0.15 ; 0.20])$ provides $\mu^{c}=207(7) \mathrm{MeV}\left(a \mu^{c}=0.118(4)\right)$ with $\chi^{2} / d o f=1.2$. This value is in agreement with our previous results for $\mu^{c}$, obtained from the $\langle q q\rangle$ fits. From Fig. 5 it is visible, that for bigger chemical potential, $\mu a>0.2$, our data deviate from the ChPT prediction.

Next, let us consider the baryon density at larger values of the chemical potential. In Fig. 6 we plot the ratio $n_{B} / n_{0}$ as a function of $\mu$, where for the square points the reference density $n_{0}$ is the baryon density for free continuum fermions at $T=0, n_{0}=\left(2 \mu^{3}\right) /\left(3 \pi^{2}\right)$, and for the circle points $n_{0}$ is the baryon number density for the free lattice fermions. It can be seen, that in the region $\mu \in[528 ; 1055]$ $\mathrm{MeV}(\mu a \in[0.3 ; 0.6])$ these ratios are slowly varying functions of the chemical potential, whereas the measured baryon density changes by an order of magnitude. We believe, that the scaling of the

\footnotetext{
${ }^{2}$ The ansatz $n_{B}(\lambda)=A+B \lambda^{2}$ was employed for the $\lambda \rightarrow 0$ extrapolation.
} 


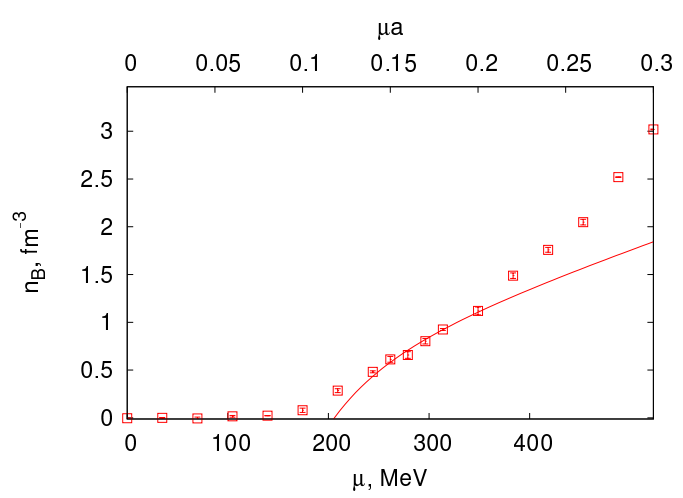

Figure 5. The baryon density $n_{B}$ in the physical units as a function of $\mu$.

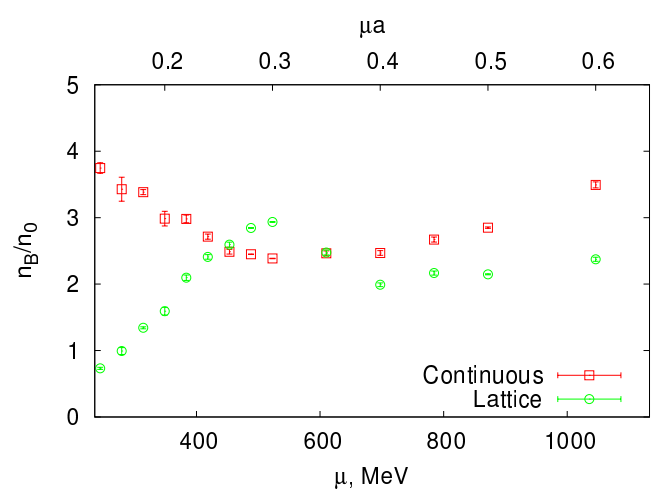

Figure 6. The ratio $n_{B} / n_{0}$ as a function of the chemical potential $\mu$.

baryon density $n_{B} \sim n_{0}$ confirms the conclusion that in the region $\mu \in[528 ; 1055] \mathrm{MeV}$ the system is in a BCS-like phase. The relevant degrees of freedom in this phase are quarks, which mostly live inside the Fermi sphere with a condensate of Cooper pairs on the Fermi surface. The fact that $n_{B} / n_{0} \sim 2.0 \ldots 2.5$, but not $\sim 1.0$, can be attributed to UV and IR effects (similar artifact effects for $n_{B}$ scaling were observed in [21]).

\subsection{The gluon observables}

In this section we study the gluon observables: Polyakov loop and Wilson loops. Similarly to the chiral condensate the gluon observables are not sensitive to the value of the $\lambda$, thus we take these observables calculated at the smallest value $\lambda=0.0005$ as their values at the $\lambda=0$.

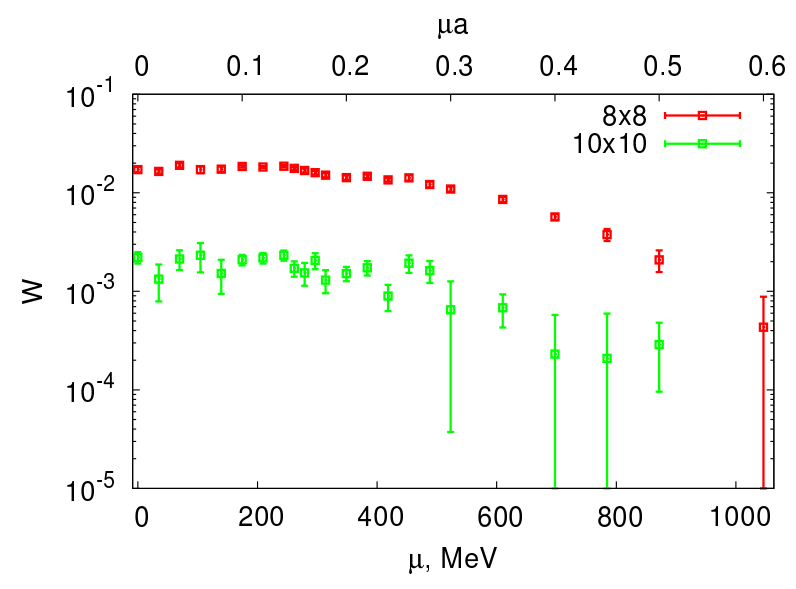

Figure 7. The time-like Wilson loops for the contours $8 \times 8$ and $10 \times 10$ as a functions of the chemical potential. 
We measured the average of the Polyakov loop as a function of the chemical potential. The result of this measurement is that for all values of the chemical potential studied in this paper the average Polyakov loop is vanishing within the uncertainty of the calculation.

Furthermore, in order to investigate the confinement properties of the system, we have calculated time-like Wilson loops for the quadratic contours of the size $8 \times 8$ and $10 \times 10$ (for larger Wilson loops we obtained results compatible with zero) as functions of the chemical potential. The results are shown in Fig. 7. One learns from this plot, that for $\mu>352 \mathrm{MeV}(\mu>0.2)$ the Wilson loops decrease with the growth of the chemical potential. At small $\mu$, for $\mu \in[0 ; 263] \mathrm{MeV}(\mu a \in[0.0 ; 0.15])$, a plateau for both Wilson loops may be noticed. From these results one can conclude, that the system is in a confined phase for all values of the chemical potential under consideration. The possible explanation for this behaviour may be the absence of the Debye screening in two-color QCD at zero temperature $[4,28]$.

\section{Discussion and conclusion}

Our results can be summarized as follows. At small chemical potential $\mu<\mu^{c}=m_{\pi} / 2 \sim 200 \mathrm{MeV}$ we observe a hadronic phase. In this phase $\mathrm{QC}_{2} \mathrm{D}$ matter is in confinement, chiral symmetry is broken, the diquark condensate vanishes and the baryon number density is also zero. Relevant degrees of freedom in this phase are Goldstone bosons.

In the region $\mu^{c}<\mu<\mu^{d} \sim 352 \mathrm{MeV}$ we observe the BEC phase. Characteristic feature of this phase is Bose-Einstein condensation of scalar diquarks. The order parameter for the transition to the BEC phase is the diquark condensate, which develops a non-zero value in the region $\mu>\mu^{c}$. Within the uncertainty of the calculation $\mu_{c}=m_{\pi} / 2$, where $m_{\pi}$ is the pion mass at zero chemical potential. In this phase, $\mathrm{QC}_{2} \mathrm{D}$ matter has also confining properties, whereas the baryon density is non-zero. Relevant degrees of freedom in the BEC phase are Goldstone bosons as well.

It is important to notice, that for all values of the chemical potential $\mu<\mu^{d}$ our results are in good agreement with the predictions of ChPT. An exception is the chiral condensate, which drops with increasing chemical potential slower than ChPT predicts in the leading order. This behaviour of the chiral condensate might be explained by higher order radiative corrections.

If we further increase the chemical potential, starting from $\mu \sim 500-600 \mathrm{MeV}$ one can observe that the diquark condensate scales as $\langle q q\rangle \propto \mu^{2}$ and the baryon density scales as $n_{B} \propto \mu^{3}$. Physically, this implies that the relevant degrees of freedom are quarks, which are mostly living inside the Fermi sphere with a condensate of Cooper pairs on the Fermi surface. These properties are clear hints in favor of the BCS phase. In this phase the chiral symmetry is restored in the chiral limit. The BCS phase extends up to $\mu \sim 1000-1100 \mathrm{MeV}$. We believe, that the BCS phase of the $\mathrm{QC}_{2} \mathrm{D}$ theory may be similar to the "quarkyonic phase" of the $\mathrm{SU}\left(N_{c}\right)$ QCD at large $N_{c}[29]$.

\section{Acknowledgments}

Numerical simulations were performed at the supercomputer of ITEP, at the federal center for collective usage at NRC "Kurchatov Institute" (http://computing.kiae.ru/) and at MSU supercomputer "Lomonosov". The work of AYK and AVM was supported by RFBR grants 14-02-01185-a, 15-0207596-a, 15-32-21117, 16-32-00048. AYK was also supported by Dynasty foundation. The work of VVB and AAN, which consisted of studying the baryon density, chiral condensate and collecting statistics, was supported by the RSF grant under contract 15-12-20008. 


\section{References}

[1] S. Muroya, A. Nakamura, C. Nonaka, T. Takaishi, Prog. Theor. Phys. 110, 615 (2003), hep-lat/0306031

[2] J.B. Kogut, M.A. Stephanov, D. Toublan, J.J.M. Verbaarschot, A. Zhitnitsky, Nucl. Phys. B582, 477 (2000), hep-ph/0001171

[3] K. Splittorff, D. Toublan, J.J.M. Verbaarschot, Nucl. Phys. B620, 290 (2002), hep-ph/0108040

[4] T. Kanazawa, T. Wettig, N. Yamamoto, JHEP 08, 003 (2009), 0906. 3579

[5] T. Brauner, K. Fukushima, Y. Hidaka, Phys. Rev. D80, 074035 (2009), [Erratum: Phys. Rev.D81,119904(2010)], 0907.4905

[6] G.f. Sun, L. He, P. Zhuang, Phys. Rev. D75, 096004 (2007), hep-ph/0703159

[7] L. He, Phys. Rev. D82, 096003 (2010), 1007.1920

[8] N. Strodthoff, B.J. Schaefer, L. von Smekal, Phys. Rev. D85, 074007 (2012), 1112 . 5401

[9] N. Strodthoff, L. von Smekal, Phys. Lett. B731, 350 (2014), 1306. 2897

[10] B. Vanderheyden, A.D. Jackson, Phys. Rev. D64, 074016 (2001), hep-ph/0102064

[11] T. Kanazawa, T. Wettig, N. Yamamoto, Phys. Rev. D81, 081701 (2010), 0912 . 4999

[12] T. Kanazawa, T. Wettig, N. Yamamoto, JHEP 12, 007 (2011), 1110. 5858

[13] T. Kanazawa, T. Wettig, N. Yamamoto, Eur. Phys. J. A49, 88 (2013), 1211.5332

[14] A. Nakamura, Phys. Lett. B149, 391 (1984)

[15] S. Hands, J.B. Kogut, M.P. Lombardo, S.E. Morrison, Nucl. Phys. B558, 327 (1999), hep-lat/9902034

[16] J.B. Kogut, D. Toublan, D.K. Sinclair, Phys. Lett. B514, 77 (2001), hep-lat/0104010

[17] J.B. Kogut, D.K. Sinclair, S.J. Hands, S.E. Morrison, Phys. Rev. D64, 094505 (2001), hep-lat/0105026

[18] J.B. Kogut, D. Toublan, D.K. Sinclair, Nucl. Phys. B642, 181 (2002), hep-lat/0205019

[19] S. Hands, S. Kim, J.I. Skullerud, Eur. Phys. J. C48, 193 (2006), hep-1at/0604004

[20] S. Hands, S. Kim, J.I. Skullerud, Phys. Rev. D81, 091502 (2010), 1001.1682

[21] S. Cotter, P. Giudice, S. Hands, J.I. Skullerud, Phys. Rev. D87, 034507 (2013), 1210. 4496

[22] T. Boz, S. Cotter, L. Fister, D. Mehta, J.I. Skullerud, Eur. Phys. J. A49, 87 (2013), 1303.3223

[23] P. Hasenfratz, F. Karsch, Phys. Lett. B125, 308 (1983)

[24] J.B. Kogut, D.K. Sinclair, Phys. Rev. D66, 034505 (2002), hep-lat/0202028

[25] J.B. Kogut, D.K. Sinclair, Phys. Rev. D70, 094501 (2004), hep-lat/0407027

[26] G. Endrödi, Phys. Rev. D90, 094501 (2014), 1407. 1216

[27] V.V. Braguta, E.M. Ilgenfritz, A.Yu. Kotov, A.V. Molochkov, A.A. Nikolaev (2016), 1605.04090

[28] D.H. Rischke, Phys. Rev. D62, 034007 (2000), nucl-th/0001040

[29] L. McLerran, R.D. Pisarski, Nucl. Phys. A796, 83 (2007), 0706. 2191 